\section{Concordance Between ' C-PIB-PET and Clinical Diagnosis in a Memory Clinic}

American Journal of Alzheimer's Disease \& Other Dementias ${ }^{\circledR}$ 20I5, Vol. 30(6) 599-606 (C) The Author(s) 2015 Reprints and permission: sagepub.com/journalsPermissions.nav DOI: $10.1177 / 1533317515576387$ aja.sagepub.com

(SAGE

\author{
Chrem Mendez Patricio, MD', Cohen Gabriela, MD', \\ Russo Maria Julieta, MD', Fernandez Suarez Marcos, MD', \\ Nahas Federico, MD', Russo Griselda, MD', Wierszylo Claudio, BA', \\ Paz Santiago, BSc', Tabaschi Leonardo, BA', Campos Jorge, MD', \\ Amengual Alejandra, MD', Kremer Janus, MD', \\ Guinjoan Salvador, MD', Leiguarda Ramon, MD', \\ Sevlever Gustavo, MD', Vázquez Silvia, MD', and Allegri Ricardo, MD'
}

\begin{abstract}
Introduction: Today, ligands that bind to fibrillar $\beta$-amyloid are detectable by Positron Emission Tomography (PET) allowing for in vivo visualization for Abeta burden. However, amyloid plaques detection per se does not establish Alzheimer's Disease diagnosis. In this sense, the utility of amyloid imaging to improve clinical diagnosis was settled only for specific clinical scenarios and few studies have assessed amyloid molecular neuroimaging in a broader clinical setting. The aim of this study is to determine the frequency of PiB amyloid findings in different diagnostic syndromes grouped into high and low probability pre- test categories, taking into account pre-test clinical assumption of the presence of AD related pathology. Methods: I44 patients were assigned into categories of high or low pretest probability according to clinical suspicion of AD pathology. The high probability group included: amnestic Mild Cognitive Impairment ( $\mathrm{MCl}$ ), amnestic and other domains $\mathrm{MCl}$, Dementia of Alzheimer's Type (DAT), Posterior Cortical Atrophy (PCA), logopenic Primary Progressive Aphasia (PPA), Cerebral Amyloid Angiopathy and mixed dementia. The low assumption group included: normal controls, non-amnestic $\mathrm{MCl}$, non-logopenic PPA and Frontotemporal Dementia (FTD). Results: Only normal controls and DAT patients (typical and atypical presentation) were the most consistent across clinical and molecular diagnostics. $\mathrm{MCl}$, non-logopenic PPA and FTD were the syndromic diagnoses that most discrepancies were found. Discussion: This study demonstrates that detecting in vivo amyloid plaques by molecular imaging is considerably frequent in most of the dementia syndromes and shows that there are frequent discordance between molecular diagnosis and clinical assumption.
\end{abstract}

\title{
Keywords
}

amyloid, neuroimaging, ' 'C-PIB-PET, memory clinic

\section{Introduction}

Amyloid plaques are a hallmark in the pathophysiology of Alzheimer's disease (AD) and constitute one of the earliest events in this disease. ${ }^{1}$ Today, ligands that bind to fibrillar $\beta$-amyloid (Abeta) are detectable by positron emission tomography (PET) allowing for in vivo visualization for Abeta burden. ${ }^{2}$ Carbon1 1-labeled Pittsburgh compound B $\left({ }^{11} \mathrm{C}-\mathrm{PIB}\right)$, a tioflavin-radiolabeled analog, was the first ligand to be developed and is the most extensively studied. There is high correlation between PIB-positive in vivo amyloid findings and autopsied amyloid cases. ${ }^{2}$ Moreover, good interreader agreement levels has shown to be reproducible. ${ }^{3}$ However, since several longitudinal studies of aging have shown that brain amyloid can be found in cognitively normal elderly people and also combined with other existing pathologies, amyloid plaques are only part of a more complex pathological mechanism and detection per se does not establish AD diagnosis. ${ }^{1}$ Therefore, the utility of amyloid imaging to improve clinical diagnosis was settled only for specific clinical scenarios. ${ }^{4,5}$ Published data on amyloid imaging usually selects patients with typical presentation, which are easier to categorize compared to the patients with routine encounter in daily practice at our memory clinic. Few studies have assessed amyloid molecular neuroimaging in a broader clinical setting. ${ }^{6}$

\footnotetext{
'Ageing and Memory Center, Instituto de Investigaciones Neurologicas "Raúl Carrea” (FLENI), Buenos Aires, Argentina

\section{Corresponding Author:}

Patricio Chrem Méndez, MD, Ageing and Memory Center, Instituto de Investigaciones Neurologicas "Raúl Carrea" (FLENI), Montañeses 2325 (CI428AQK), Buenos Aires, Argentina.

Email: patriciochrem@gmail.com
} 
The aim of this study is to determine the frequency of PIB amyloid findings in different diagnostic syndromes grouped into high and low probability pretest categories, taking into account pretest clinical assumption of the presence of AD-related pathology. Then we compare concordance with molecular diagnosis in each syndromic diagnosis. Finally, we reviewed some potential clinical consequences regarding PET amyloid scans in clinical setting.

\section{Material and Method}

\section{Study Population and Baseline Evaluations}

This is an observational retrospective study designed to describe our initial experience with the use of amyloid PET imaging in routine daily patients seen in an Aging and Memory Center, Neurological Research Institute (FLENI), Buenos Aires, Argentina. We included for analysis the first 178 patients who underwent ${ }^{11} \mathrm{C}$-PIB-PET in our center from December 2012 to December 2013. Since we are a referral center for cognitive disorders, patients of our clinic tend to have atypical rather typical presentation. In all, 40 of these patients belong to the study protocol Argentina-Alzheimer's Disease Neuroimaging Initiative (ADNI Arg); 13 patients were referred for the PET test from the Kremer's Neuropsychiatric Institute in Córdoba, Argentina; the rest of the patients were either referred for PET test from our Aging and Memory Center or were inpatients from the Neurology Department from FLENI. Thirtyfour patients who were not evaluated by our team or who lacked the baseline neuropychological or neuropsychiatric assessment were excluded from this analysis. Finally, 144 patients were included for analysis.

All patients had a standard baseline evaluation performed by a doctor experienced in memory disorders (neurologists, psychiatrists, or geriatricians) that included a medical interview, informant-based clinical history taking, physical, and neurologic examination. Performed blood tests to rule out reversible causes of dementia included basic chemistry, cell blood count, calcium, thyroid test, HIV serology, levels of vitamin B12, folic acid, and homocysteine levels. Furthermore, neuropsychological assessment was performed by a neuropsychologist and included:

- $\quad$ Mini-Mental State Examination (MMSE) ${ }^{7}$

- Wechsler logical memory (WMT), ${ }^{8}$

- Rey auditory verbal learning test (RAVLT), ${ }^{9}$

- Boston naming test, ${ }^{10}$

- Semantic and phonological fluency for language evaluation, ${ }^{11}$

- Direct and reverse digit span, ${ }^{12}$

- Trail making test (TMT), ${ }^{13}$ and

- Frontal assessment battery (FAB) for executive function. ${ }^{14}$

All patients also underwent magnetic resonance imaging (MRI) of the brain including T1, T2, fluid-attenuated inversion recovery, and gradient-recalled echo, and diffusion-weighted imaging sequences and images were saved in Kodak Carestream System from FLENI for clinical reviewing.

\section{Patient's Classification Based on Clinical Data}

Based on pre-PET results obtained, patients were classified by dementia experts into the following groups:

1. Normal controls

2. Amnestic-type mild cognitive impairment (a-MCI): patients with memory complaint with only abnormal $Z$ score below 1.5 standard deviation (SD) from the mean (matched by age and sex) in the memory tests $\left(\mathrm{WMT}^{8}\right.$ and $\mathrm{RAVLT}^{9}$ ) without functional impairment.

3. Amnestic multidomain mild cognitive impairment (am-MCI): patients with memory complaint and alteration in other domains with a $Z$ score below 1.5 $\mathrm{SD}$, without functional impairment.

4. Nonamnestic mild cognitive impairment (nonamnesticMCI): patients with any cognitive complaint without evidence of memory impairment but with significant impairment in other cognitive domains with a $Z$ score below 1.5 SD. They had no functional impairment.

5. Dementia of Alzheimer's type (DAT): based on probable Alzheimer's Dementia National Institute of Neurological and Communicative Disorders and Stroke Alzheimer's Disease and Related Disorders Association (NINCDS-ADRDA) clinical criteria. ${ }^{15}$

6. Behavioral variant of frontotemporal dementia based on the Rascovsky et al criteria ${ }^{16}$

7. Primary progressive aphasia (PPA): patients with language alterations based on Mesulam PPA criteria ${ }^{17}$ and its variants according Gorno-Tempini et al criteria ${ }^{18}$

8. Cortical posterior atrophy: patients with visual spatial and parietal alterations based on a revision from Crutch et al criteria. ${ }^{19}$

9. Mixed dementia: based on probable DAT defined by NINCDS-ADRDA criteria and evidence of cerebrovascular pathology in brain MRI.

10. Other degenerative disorders including 2 patients with clinical and neuroimaging finding of probable cerebral amyloid angiopathy (CAA) according to the Boston criteria $^{20}$; and 1 patient fulfilled clinical features for corticobasal syndrome (CBS).

Based on their respective physiopathologies and using their possible relation to amyloid deposits as the main variable, all 9 aforementioned disease categories were assigned into groups of either high or low pretest amyloid deposit probability. Within the high probability group, we included patients in the a-MCI, am-MCI, DTA, PCA, CAA, logopenic variant of PPA, and mixed dementia group. The low probability group comprised the control group and patients with clinical diagnosis of nonamnestic MCI, bvFTD, nonlogopenic-PPA (semantic variant and nonfluent agrammatic aphasia), and CBS. 
Table I. Demographic Data of Diagnostic Groups. ${ }^{\text {a }}$

\begin{tabular}{|c|c|c|c|c|c|c|c|c|}
\hline & \multirow[b]{2}{*}{$\mathrm{n}$} & \multicolumn{2}{|c|}{ Age at Scan } & \multicolumn{2}{|c|}{ Education } & \multicolumn{2}{|c|}{ MMSE } & \multirow[b]{2}{*}{ Gender, Female, \% (n) } \\
\hline & & Mean & SD & Mean & SD & Mean & SD & \\
\hline Controls & 17 & 63.35 & 6.86 & 15.94 & 2.57 & 29.63 & 0.89 & $64.7(\mathrm{II})$ \\
\hline $\mathrm{a}-\mathrm{MCl}$ & 32 & 69.97 & 7.69 & 14.07 & 4.63 & 27.31 & 2.80 & $53.1(17)$ \\
\hline am-MCl & 20 & 70.35 & 7.73 & 14.77 & 3.90 & 27.24 & 3.05 & $65.0(13)$ \\
\hline Nonmemory-MCl & 18 & 67.11 & 5.56 & 13.67 & 3.12 & 28.19 & 1.60 & $27.8(5)$ \\
\hline DAT & 21 & 70.14 & 5.78 & 13.37 & 3.45 & 23.29 & 4.01 & $66.7(14)$ \\
\hline bvFTD & 6 & 61.60 & 14.36 & 15.00 & 4.24 & 23.00 & 9.54 & $66.7(4)$ \\
\hline PPA & II & 61.27 & 10.10 & 14.60 & 4.51 & 24.43 & 4.39 & $54.5(6)$ \\
\hline Logopenic PPA & 4 & 67.25 & 5.25 & 13.50 & 2.12 & 24.50 & 2.12 & $75.0(3)$ \\
\hline PCA & 5 & 65.00 & 11.25 & 13.00 & 4.55 & 23.00 & 7.52 & $40.0(2)$ \\
\hline Amyloid angiopathy & 2 & 66.50 & 3.54 & 9.00 & 0.10 & 13.00 & 0.10 & $0(0)$ \\
\hline Mixed dementia & 7 & 70.43 & 6.11 & 14.60 & 2.61 & 21.40 & 6.39 & 57.1 (4) \\
\hline CBD & I & 70.00 & & & & 24.00 & & $100(1)$ \\
\hline
\end{tabular}

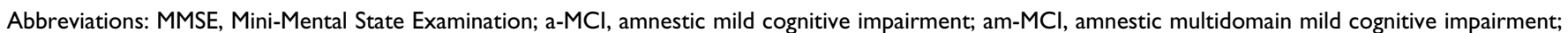

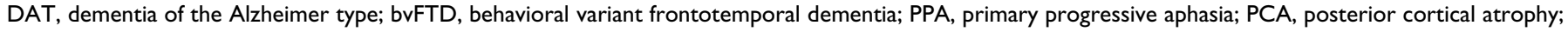
CBD, corticobasal degeneration.

${ }^{\mathrm{a}}$ All values are means and standard deviations (SDs), or percentages.

\section{Synthesis and Purification of " C-PIB-PET}

The synthesis of ${ }^{11} \mathrm{C}$-PIB is performed in the Tracer LAB FXCPRO (General Electric RIS (Radiology Information System) Carestream); the process' duration from the reception of ${ }^{11} \mathrm{C}$ $\mathrm{CO}_{2}$ takes approximately 25 minutes and another 10 minutes for the purification. The purification is performed through the differential interaction among the components of the row product, the stationary phase, and the mobile phase. The final purification is performed within the synthesis module. The final result is a mixed solution of the mobile phase with a volume of $28 \mathrm{~mL}$ of physiologic solution with a content of less than $10 \%$ of ethanol and $200 \mathrm{mCi}$ approximately of ${ }^{11} \mathrm{C}$-PIB. The final result of the reaction is of $10 \%$.

At 50 minutes of the endovenous infusion of $10 \mathrm{mCi}$ of ${ }^{11} \mathrm{C}$-PIB (Pittsburgh Component) volumetric multislice computerized tomography (CT) brain images were obtained using PET/CT Discovery 690 GE equipment (General Electric RIS (Radiology Information System) Carestream). Images were obtained using a nonuniform attenuation correction with CT. Axial, coronal, and sagittal images were obtained either with MRI and/or CT.

\section{Visual Analysis of PET}

Images were viewed and analyzed by 2 nuclear medicine physicians, blinded to the clinical data of the patients. Both readers are experienced in ${ }^{11} \mathrm{C}$-PIB interpretation as they are currently part of the PET team of the ongoing ADNI Arg since $2011 .^{21}$ The presence or absence of cortical brain amyloid and its spatial distribution was analyzed in a qualitative way using visual color linear scales. Based on the concentration of activity of ${ }^{11} \mathrm{C}$-PIB, the degree of cortical retention was classified as positive or negative. The interobserver variability was $5 \%$. No other semiquantitative or quantitative assay was performed from the PET images.

\section{Statistic Analysis}

The SPSS Statistical package (version 19.0) was used for the data analyses. Demographic variables (age and education) and neuropsychological battery scores were compared by the Student's $t$ test. Pearson's chi-square test was employed for categorical data (gender). Assumption of variance homogeneity was assessed using Levene's test. In order to investigate the effects of age and education, we conducted an analysis of covariance (ANCOVA) adjusted for age and education in years followed by Bonferroni's post hoc analysis. Neuropsychological test battery scores were assessed by the raw scores of all included tests. We applied the Spearman's correlation 2-tailed analysis test to investigate the relation between the demographic variables and neuropsychological-biological data. Effect size was calculated using $\varphi$ coefficient for the Pearson's chi-square test. A $P$ level of $<.05$ was considered as statistically significant level.

The $2 \times 2$ contingency analysis was used to assess the accuracy of the ${ }^{11} \mathrm{C}$-PIB-PET visual interpretation when used to distinguish high from low pretest probability and to determine the test sensitivity and specificity. In order to assess the effect of age on diagnostic accuracy, the cohort was split into 2 groups according to the median age of the sample, with younger ( $\leq 68$ years) and older ( $>68$ years). The above-mentioned analyses were performed on both categories. Finally, we compared differences in clinical and neuropsychological variables in the $\mathrm{PIB}+$ to PIB - groups.

\section{Results}

Table 1 shows the sociodemographic characteristics and MMSE scores of the participants, stratified by clinical diagnosis. Clinical diagnoses were divided into high or low pretest probability categories based on the association of the clinical syndrome with predicted histopathology. Sociodemographic characteristics and neuropsychological test battery results for 
Table 2. Demographic and Clinical Data of the Low and High Pretest Probability Groups. ${ }^{a}$

\begin{tabular}{|c|c|c|c|c|c|c|}
\hline & \multicolumn{2}{|c|}{$\begin{array}{l}\text { Low Pretest } \\
\text { Probability }\end{array}$} & \multicolumn{2}{|c|}{$\begin{array}{l}\text { High Pretest } \\
\text { Probability }\end{array}$} & \multicolumn{2}{|c|}{-Student's $t$} \\
\hline & Mean & SD & Mean & SD & $t$ & $P$ \\
\hline Age & 65.89 & 8.35 & 69.66 & 7.18 & -4.17 & $<.001$ \\
\hline Gender (male/female) & \multicolumn{2}{|c|}{$26 / 27$} & \multicolumn{2}{|c|}{$38 / 53$} & 0.723 & .487 \\
\hline Years of evolution & 2.02 & 1.79 & 2.63 & 2.12 & -1.60 & .11 \\
\hline Education & 14.73 & 3.19 & 13.90 & 3.97 & 1.14 & .26 \\
\hline MMSE & 27.65 & 3.67 & 25.46 & 4.51 & $2.7 I$ & .01 \\
\hline RAVLT total & 35.56 & 10.60 & 26.77 & 8.64 & 4.35 & $<.001$ \\
\hline RAVLT delayed & 5.64 & 3.59 & 1.86 & 2.56 & 5.98 & $<.001$ \\
\hline RAVLT recognition & 11.09 & 3.15 & 8.11 & 4.25 & 3.54 & $<.00$ \\
\hline Category fluency test & 16.86 & 5.50 & 15.21 & 5.38 & 1.47 & .14 \\
\hline $\begin{array}{l}\text { Phonological fluency } \\
\text { test }\end{array}$ & 13.20 & 5.78 & 12.57 & 5.88 & 0.51 & .60 \\
\hline Forward digit span & 5.90 & 1.16 & 5.70 & 1.30 & 0.74 & .46 \\
\hline Backward digit span & 3.87 & 0.88 & 3.29 & 1.13 & 2.52 & .01 \\
\hline TMT-A & 47.29 & 25.69 & 76.19 & 65.18 & -2.52 & .01 \\
\hline TMT-B & 111.25 & 71.83 & 157.78 & 85.72 & -2.58 & .01 \\
\hline GDS & 3.21 & 3.01 & 2.48 & 2.18 & 1.19 & .24 \\
\hline NPI-Q & 7.39 & 7.24 & 5.04 & 4.74 & 1.55 & .12 \\
\hline FAQ & 5.67 & 4.90 & 6.76 & 6.99 & -0.60 & .55 \\
\hline
\end{tabular}

Abbreviations: MMSE, Mini-Mental State Examination; RAVLT, rey auditory verbal learning test; TMT-A, trail making test part $A$; TMT-B, trail making test part B; GDS, geriatric depression scale; NPI-Q, neuropsychiatric inventory questionnaire; $F A Q$, functional activities questionnaire.

${ }^{a} A$ All values are means and standard deviations (SDs), or percentages. Pearson's chi-square test. Student's $t$ test, 2-tailed, and unequal variance.

both groups are listed in Table 2 . Both groups were similar with respect to gender $\left(\chi^{2}=0.723, P=.487\right)$, years of education $(t=1.14, P=.26)$, and years of disease evolution $(t=$ $-1.60, P=.11)$. Compared to the low pretest probability group, the high pretest probability patients were older $(t=$ -4.17, $P<.001)$, had lower MMSE $(t=4.35, P=.01)$, RAVLT total $(t=4.35, P<.001)$, RAVLT delayed $(t=$ 5.98, $P<.001)$ and RAVLT recognition $(t=3.54, P<.001)$, backward digit span $(t=2.52, P=.01)$, TMT-A $(t=-2.52$, $P=.01)$, and TMT-B $(t=-2.58, P=.01)$ scores. Covarying for age or education had no effect on these results (ANCOVA results not shown).

Positive ${ }^{11} \mathrm{C}$-PIB scans by visual inspection was positively correlated with age $(P=.17)$ and negatively with years of education $(P<.001)$, MMSE, RAVLT total, delayed and recognition scores $(P<.001)$, category fluency test $(P=.002)$, TMT-A $(P<.001)$, and TMT-B $(P=.001)$.

We include 144 patients. In all, 63\% (91 of 144) was assigned to the high pretest probability subgroup (Table 3), and $37 \%$ (53 of 144) was assigned to the low pretest probability subgroup (Table 4) based on the clinical probability of having an underlying AD neuropathology.

Overall concordance between scan results and clinical diagnosis was $72.6 \%$ for high pretest probability and $73.6 \%$ for low pretest probability. Of the high pretest probability patients, $68.8 \%$ (22 of 32) a-MCI, $60 \%$ (12 of 20) am-MCI, $76.2 \%$ (16 of 21) DAT, all the patients who had Logopenic-PPA (4
Table 3. Results of ' $\mathrm{C}-\mathrm{PIB}$ Scans by Visual Inspection in Patients With High Pretest Probability of Having an Underlying AD Neuropathology.

\begin{tabular}{lrrr}
\hline & & \multicolumn{2}{c}{$\mathrm{PIB}+$} \\
\cline { 3 - 4 } Diagnosis & $\mathrm{n}$ & $\mathrm{n}$ & $\%$ \\
\hline a-MCl & 32 & 22 & 68.8 \\
am-MCl & 20 & 12 & 60.0 \\
DAT & 21 & 16 & 76.2 \\
Logopenic PPA & 4 & 4 & 100.0 \\
PCA & 5 & 5 & 100.0 \\
Amyloid angiopathy & 2 & 2 & 100.0 \\
Mixed dementia & 7 & 5 & 71.4 \\
\hline
\end{tabular}

Abbreviations: a-MCl, amnestic mild cognitive impairment; am- $\mathrm{MCl}$, amnestic multidomain mild cognitive impairment; DAT, dementia of Alzheimer's type; PPA, primary progressive aphasia; PCA, posterior cortical atrophy; PIB, Pittsburgh compound B; AD, Alzheimer's disease.

Table 4. Results of " 'C-PIB Scans by Visual Inspection in Patients with Low Pretest Probability of Having an Underlying AD Neuropathology.

\begin{tabular}{lrrc}
\hline & & \multicolumn{2}{c}{ PIB +} \\
\cline { 3 - 4 } Diagnosis & $\mathrm{n}$ & $\mathrm{n}$ & $\%$ \\
\hline Controls & 17 & 1 & 5.0 \\
Nonmemory-MCl & 18 & 6 & 33.0 \\
bvFTD & 6 & 2 & 33.0 \\
PPA & 11 & 5 & 45.0 \\
CBD & 1 & 0 & 0
\end{tabular}

Abbreviations: bvFTD, behavioral variant frontotemporal dementia; PPA, primary progressive aphasia; $\mathrm{CBD}$, corticobasal degeneration; PIB, Pittsburgh compound $\mathrm{B} ; \mathrm{AD}$, Alzheimer's disease; $\mathrm{MCl}$, mild cognitive impairment.

of 4) and PCA (5 of 5), both cases of CAA (2 of 2), and $71.4 \%$ (5 of 7 ) patients with mixed dementia had positive ${ }^{11} \mathrm{C}$-PIB scans by visual inspection. By contrast, the low pretest probability group was more heterogeneous. In all, 5\% (1 of 17) control patients, $33 \%$ (6 of 18) nonmemory-MCI, $33 \%$ (2 of 6 ) bvFTD, and $45 \%$ (5 of 11) patients with PPA showed positive ${ }^{11} \mathrm{C}$-PIB scans. Figure 1 shows the percentage of concordance between clinical diagnosis and ${ }^{11} \mathrm{C}$-PIB PET results.

Our analyses indicated that a positive ${ }^{11} \mathrm{C}-\mathrm{PIB}(72.5 \%$ vs $\left.26.4 \%, \chi^{2}=28.843, P<.001\right)$ was more likely within the high probability group than the low probability group. These results did not change when young and older groups were analyzed separately. Our analyses also indicated that in our sample there is a significant association between pretest probability and ${ }^{11} \mathrm{C}$-PIB result $(\varphi=.448, d f=1, P<.0001)$.

The test sensitivity, specificity, and accuracy of visual interpretation of ${ }^{11} \mathrm{C}$-PIB are summarized in Table 5. The visual interpretation of ${ }^{11} \mathrm{C}$-PIB showed sensitivity (0.72), specificity (0.73), and accuracy ( 0.72$)$ to differentiate between high from low pretest probability. Age appeared to have an impact on sensitivity but not on specificity or accuracy in ${ }^{11} \mathrm{C}-\mathrm{PIB}$. A reduction in sensitivity was observed in the younger group when compared with the older patients. 


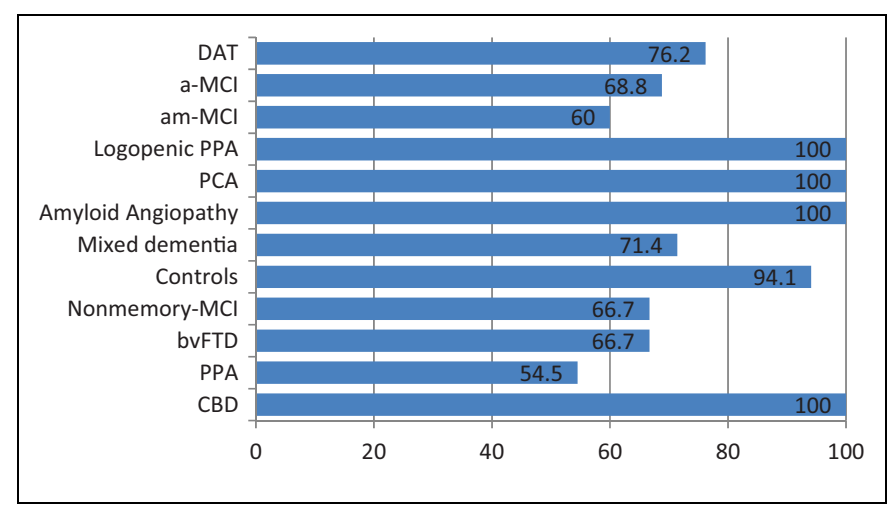

Figure I. Percentage of concordance between clinical diagnosis and carbon I I-labeled Pittsburgh compound B ( ' I C-PIB-PET) results. DAT indicates dementia of the Alzheimer type; a-MCl, amnestic mild cognitive impairment; am- $\mathrm{MCl}$, amnestic multidomain mild cognitive impairment; bvFTD, behavioral variant frontotemporal dementia; PPA, Primary progressive aphasia; PCA, posterior cortical atrophy; $\mathrm{CBD}$, corticobasal degeneration.

Table 5. Sensitivity, Specificity, and Accuracy of ${ }^{1 /} \mathrm{C}-\mathrm{PIB}$ (Visual Analysis).

\begin{tabular}{lccc}
\hline & Sensitivity & Specificity & Accuracy \\
\hline High vs low pretest probability & 0.72 & 0.73 & 0.72 \\
Young $(\leq 68$ y) & 0.68 & 0.75 & 0.72 \\
Old $(>68$ y) & 0.75 & 0.73 & 0.75 \\
\hline
\end{tabular}

Abbreviation: PIB, Pittsburgh compound B.

\section{Discussion}

In the present study, we described findings of brain amyloid deposition using ${ }^{11} \mathrm{C}$-PIB-PET in a large sample of routine patients diagnosed with cognitive prevalent syndromes consulting at a memory clinic in Buenos Aires. In general, elevated frequency of positive PIBs was detected in the high probability pretest group and elevated frequency of negative PET studies was observed in the low pretest probability group. However, considerable differences were evident between clinical diagnosis and results of molecular imaging in both groups. The sample was negative in $29.67 \%$ of the patients in the high probability group, whereas it was positive in $26.42 \%$ of patients in the low probability, in which amyloid deposition was not expected. When calculating sensitivity and specificity according to high and low pretest probability, PIB sensitivity was lower in younger when compared to older ones, whereas specificity remains the same (Table 4). Other studies have shown discrepancy between clinical and molecular diagnosis of almost $39 \% .{ }^{5}$ These results that significantly contradict the initial clinical assumption may entail consequences in relation to patient management. Given that these consequences may be different in patients from both low and high pretest probability groups, it is important to analyze concordances and implications for each syndromic category.

Patients diagnosed with a-MCI or am-MCI had the lowest rates of concordance when compared to molecular diagnosis in the high pretest probability group $(68.8 \%$ and $60 \%$, respectively). According to the new diagnostic recommendations, an intermediate risk of MCI due to AD exists in patients in whom brain amyloid was detected. Higher risk is conferred to patients with neurodegenerative markers such as $\tau$ in CSF, MRI hippocampal atrophy, and fludeoxyglucose-PET AD signature, when added to amyloid detection. ${ }^{22}$ However, PIB did predict progression to $\mathrm{AD}$ in longitudinal studies in patients with $\mathrm{MCI}$ without neurodegeneration markers. ${ }^{23}$ Some studies consider a-MCI and positive PIB scenario as prodromal stage of $\mathrm{AD} .{ }^{24}$ Finally a-MCI and am-MCI have increased AD conversion risk when combined with positive PIB. Preventive strategies on risk factor control should be emphasized for this group when considering the additional value of molecular imaging. ${ }^{25}$

More interesting are patients with a-MCI having negative PIB (31.2\%). According to current diagnostic criteria recommendations, this group has a lower risk of $\mathrm{AD}$ conversion. ${ }^{22}$ Considering amyloid deposition occurs between 10 and 20 years before clinical manifestations, ${ }^{1}$ these patients have a very low probability of developing AD. Patients with MCI having negative PIB correspond to a scenario that reinforces the need to search for other pathologies. Further tests and treatments on them are unnecessary and should be avoided. In this regard, the US Food and Drug administration has recently accepted the negative predictive value of fluorine-based amyloid tracer florbetapir ${ }^{26,27}$ (which has a longer half-life and allowed its commercialization). In this context, PIB was studied more than florbetapir, with the same negative predictive value applying to nonamnestic patients with MCI having negative PIB. On the other hand, positive PIB in patients with nonamnestic MCI does not improve diagnostic certainty. Although the risk of conversion to AD is increased, ${ }^{28}$ other non-AD pathologies cannot be ruled out.

In the DAT group, $76 \%$ of these patients had positive PIB. In this category, there is a high correlation between clinical and molecular diagnosis. Perhaps clinical uncertainty at this stage of disease is low, making molecular diagnosis redundant. ${ }^{2}$ Five (23.8\%) patients clinically diagnosed as having AD had negative PIB scans. In these patients, the possibility of a falsè-negative result could not be ruled out. ${ }^{29}$ However, non-AD pathology in patients with AD-like phenotype is not uncommon. ${ }^{30}$ Furthermore, AD-like phenotype was found in patients with mutations in genes implicated in FTD (c9orf72). ${ }^{31}$ In our sample, age of patients with AD was lower and MMSE average performance was higher compared to other studies. Most patients with $\mathrm{AD}$ were at a mild stage of the disease and part of ADNI-Arg. In the rest of the cases with DAT, PIB was performed in early-onset cases. As our DAT sample corresponded to mild and early-onset cases, not many differences were found compared with the percentage of molecular agreement found in aMCI $(68.8 \%)$. Fewer requests for molecular imaging were ordered in patients with more advanced disease, since molecular diagnosis does not change patient perspective. We concluded there is strong pretest presumption of amyloid deposition in the $\mathrm{AD}$ group and positive PIB may reinforce initial diagnosis in patients with AD. However, we consider PIB clinically useful mainly in early-onset patients so as to rule out 
alternative diagnoses. Also, a negative amyloid scan may have consequences in medication management, although no study to date has assessed the value of antidementials in amyloid negative patients. Same considerations regarding patients with mixed dementia apply, as they were defined as patients with DAT with evidence of cerebrovascular disease and were included in the high pretest probability group. In fact, these patients have shown similar concordance rate to patients with DAT (71.4\%).

Regarding the other patients included in the high pretest probability group (PCA, logopenic-PPA, and CAA), few conclusions can be drawn due to the small sample recruited. In these patients, however, mean age was substantially low since all cases were presenile onset. All patients had positive amyloid findings (maximum concordance). However, other differential diagnoses of young onset dementia should also be considered (FTD, Lewy's body disease, and CreutzfeldtJakob's disease). ${ }^{32}$ Establishing diagnosis of one of the latter may have significant prognostic, therapeutic, and genetic implications. Thus, presence or absence of amyloid in young patients with atypical variants may be especially useful for diagnosis and have particular impact on medical decision making.

In patients included in the frontotemporal lobar degeneration spectrum (which is considered a low probability pretest group), results were heterogeneous with the lowest concordance rates between categories. Two (33\%) patients with behavioral variant FTD (bvFTD) had a positive amyloid scan. ${ }^{33}$ These cases raise 2 possibilities: frontal variant $\mathrm{AD}^{34}$ or coexisting pathology (amyloid deposits in a patient with pathological FTD). The remaining patients with bvFTD had negative PIB scans. As mentioned previously, presence of AD biomarkers represented an exclusion criterion for Rascovsky et al. ${ }^{33}$ In patients with nonfluent/agrammatic or semantic variants of PPA, presence of amyloid was unexpected $(80 \%)$. Although pathology finding overlap is usually found in PPA, the overlap percentage is different for each variant: In nonfluent/agrammatic PPA, AD pathology is found in $30 \%$ of autopsied patients, and in the semantic variant, in less than $10 \% .^{35}$ Furthermore, clinical distinction between PPA variants may be difficult in some patients, especially between nonfluent/ agrammatic and logopenic variants. ${ }^{36,37}$ In this sense, and in addition to linguistic considerations, PIB might be useful to identify true underlying pathology.

Finally, normal controls were incorporated into the low probability pretest group. Almost all of them were part of ADNI Arg. Only 1 of the 17 participants presented had a positive amyloid scan. Other articles reported that when PIB scans are conducted in normal volunteers, $10 \%$ to $30 \%$ had positive amyloid scans. ${ }^{38}$ The frequencies shown in these studies were related to age and apolipoprotein E (APOE) genotype. ${ }^{39}$ The mean age of our normal control group was the lowest of the sample (APOE genotyping is still in process). Significance of amyloid in normal volunteers remains uncertain and with no appropriate indication to order PIB in normal cognitive participants up to date. So far, the limited longitudinal studies on the patient show little agreement. ${ }^{39,40}$ Although these patients may correspond to an AD preclinical stage (stage 1$),{ }^{41}$ studies have shown that progression to $\mathrm{AD}$ is more related to neurodegenerative markers. ${ }^{42}$ Only 1 patient presented with parkinsonism (CBS). The small number of patients recruited could respond to the fact that several studies have shown frequent co-occurrence of amyloid plaques in most of the extrapyramidal syndromes. ${ }^{12,43}$ The lack of specificity reported in these particular types of patients probably makes specialists avoid amyloid neuroimaging. Moreover, CBS has also been described in relation to $\mathrm{AD}$ pathology. ${ }^{44,45}$

The present study has some limitations. This is a descriptive study that only assesses amyloid detection frequencies in different clinical syndromes. Sample selection was performed in consecutive way; therefore, few patients were recruited for some clinical syndromes and not every clinical scenario was contemplated. As patients could not be matched on sex, age, and educational level, this led to differences in amyloid frequencies when compared to others studies. Especially, in the $\mathrm{CN}$ group which had the lowest mean age and, as it was mentioned previously, amyloid findings in this group are related to age and APOE status. ${ }^{38}$ In comparison with other ${ }^{18}$ Fluor-labeled tracers studies, no quantitative image analysis was made in this study (standardized uptake values are used for normalizing injected dose and body weight). ${ }^{27,46}$ None of the patients has undergone brain autopsy for etiological confirmation.

To conclude, it may be difficult to diagnose patients with dementia syndromes based solely on clinical data due to overlap between distinct clinical syndrome and findings in neuropathology. This study demonstrates that detecting in vivo amyloid plaques by molecular imaging is considerably frequent in most of the dementia syndromes. It further shows that there are frequent discordance between molecular diagnosis and clinical assumption. There is thus a significant need to improve diagnostic accuracy. The PIB offers additional data about the possible underlying mechanisms but not enough to establish AD diagnosis. The additional value of PIB in the diagnostic process is different for each clinical syndrome. We strongly believe amyloid detection may have significant impact in the clinical setting of early AD onset (prodromal or clinical stages) and for atypical features such as PCA and logopenicPPA variant. For the remaining categories described (bvFTD, nonlogopenic PPA, and nonamnestic MCI), concordance rates are lower and a positive amyloid scan does not establish cause or coexistence of pathology.

\section{Acknowledgments}

Bioingeneers: German Falasco, Leandro Leikis and Federico Diaz, Physicist: Leandro Urrutia, Technicians. Carla Mencia, Gonzalo Calero and Luciano Vazquez.

\section{Declaration of Conflicting Interests}

The authors declared no potential conflicts of interest with respect to the research, authorship, and/or publication of this article. 


\section{Funding}

The authors received no financial support for the research, authorship, and/or publication of this article.

\section{References}

1. Jack CR, Knopman DS, Jagust WJ, et al. Hypothetical model of dynamic biomarkers of the Alzheimer's pathological cascade. Lancet Neurol. 2010;9(1):119-128. http://www.pubmedcentral. nih.gov/articlerender.fcgi? artid $=2819840 \&$ tool $=$ pmcentrez\& rendertype $=$ abstract (accessed February 21, 2014).

2. Ikonomovic MD, Klunk WE, Abrahamson EE, et al. Post-mortem correlates of in vivo PiB-PET amyloid imaging in a typical case of Alzheimer's disease. Brain. 2008;131(pt 6):1630-1645. http:// www.pubmedcentral.nih.gov/articlerender.fcgi?artid=2408940\& tool $=$ pmcentrez\&rendertype $=$ abstract $\quad$ (accessed February 28, 2014).

3. Tolboom N, van der Flier WM, Boverhoff J, et al. Molecular imaging in the diagnosis of Alzheimer's disease: visual assessment of [11C]PIB and [18F]FDDNP PET images. J Neurol Neurosurg Psychiatry. 2010;81(8):882-884. http://www.ncbi.nlm.nih.gov/ pubmed/20543188 (accessed March 9, 2014).

4. Johnson KA, Minoshima S, Bohnen NI, et al. Appropriate use criteria for amyloid PET: a report of the Amyloid Imaging Task Force, the Society of Nuclear Medicine and Molecular Imaging, and the Alzheimer's Association. Alzheimers Dement. 2013;9(1): e-1-16. http://www.pubmedcentral.nih.gov/articlerender. fcgi? artid $=3733252 \&$ tool $=$ pmcentrez $\&$ rendertype $=$ abstract $($ accessed March 9, 2014).

5. Ossenkoppele R, Prins ND, Pijnenburg Y, et al. Impact of molecular imaging on the diagnostic process in a memory clinic. Alzheimers Dement. 2013;9(4):414-421. http://www.ncbi.nlm.nih.gov/ pubmed/23164552 (accessed March 9, 2014).

6. Wolk DA. Amyloid imaging in atypical presentations of Alzheimer's disease. Curr Neurol Neurosci Rep. 2013;13(12): 412. http://www.ncbi.nlm.nih.gov/pubmed/24136459 (accessed March 9, 2014).

7. Folstein MF, Folstein SE, McHugh PR. "Mini-mental state". A practical method for grading the cognitive state of patients for the clinician. J Psychiatr Res. 1975;12(3):189-198. http://www.ncbi. nlm.nih.gov/pubmed/1202204 (accessed March 9, 2014).

8. Wechsler D. Wechsler Memory Scale. New York: The Psychological Corporation; 1987.

9. Schoenberg MR, Dawson KA, Duff K, Patton D, Scott JG, Adams RL. Test performance and classification statistics for the Rey Auditory Verbal Learning Test in selected clinical samples. Arch Clin Neuropsychol. 2006;21(7):693-703. http://www.ncbi.nlm. nih.gov/pubmed/16987634 (accessed January 27, 2014).

10. Serrano C, Allegri RF, Drake M, et al. A shortened form of the Spanish Boston naming test: a useful tool for the diagnosis of Alzheimer's disease [in Spanish]. Rev Neurol. 2001;33(7): 624-647. http://www.ncbi.nlm.nih.gov/pubmed/11784949 (accessed March 9, 2014).

11. Morris JC, Heyman A, Mohs RC, Hughes JP, van Belle G, Fillenbaum G. The consortium to establish a registry for Alzheimer's disease (CERAD). Part I. Clinical and neuropsychological assessment of Alzheimer's disease. Neurology. 1989;39(9): 1159-1165.

12. Wechsler D. Wechsler Memory Scale. 3rd ed. San Antonio: TX Psychol Corp; 1997.

13. Tombaugh TN. Trail making test A and B: normative data stratified by age and education. Arch Clin Neuropsychol. 2004;19(2):2032014. http://www.ncbi.nlm.nih.gov/pubmed/15010086 (accessed January 30, 2014).

14. Dubois BSAPBLI. The FAB: a frontal assessment battery at bedside. Neurology. 2000;55(11):1613-1620.

15. McKhann GM, Knopman DS, Chertkow H, et al. The diagnosis of dementia due to Alzheimer's disease: recommendations from the National Institute on Aging-Alzheimer's association workgroups on diagnostic guidelines for Alzheimer's disease. Alzheimers Dement. 2011;7(3):263-269. http://www.pubmedcentral.nih.gov/ articlerender.fcgi?artid $=3312024 \&$ tool $=$ pmcentrez\&rendertype $=$ abstract (accessed November 8, 2013).

16. Rascovsky K, Hodges JR, Knopman D, et al. Sensitivity of revised diagnostic criteria for the behavioural variant of frontotemporal dementia. Brain. 2011;134(pt 9):2456-2477. http:// www.pubmedcentral.nih.gov/articlerender.fcgi?artid $=3170532 \&$ tool $=$ pmcentrez\&rendertype $=$ abstract $\quad($ accessed November 7 , 2013).

17. Mesulam MM. Primary progressive aphasia-a language-based dementia. $N$ Engl J Med. 2003;349(16):1535-1542. http:// www.ncbi.nlm.nih.gov/pubmed/14561797 (accessed October 16, 2003).

18. Gorno-Tempini ML, Hillis a E, Weintraub S, et al. Classification of primary progressive aphasia and its variants. Neurology. 2011;76(11):1006-1014. http://www.pubmedcentral.nih.gov/article render.fcgi? artid $=3059138 \&$ tool $=$ pmcentrez\&rendertype $=$ abstract (accessed March 15, 2011).

19. Crutch SJ, Lehmann M, Schott JM, Rabinovici GD, Rossor MN, Fox NC. Posterior cortical atrophy. Lancet Neurol. 2012;11(2):170-178. http://www.pubmedcentral.nih.gov/article render.fcgi? artid $=3740271 \&$ tool $=$ pmcentrez\&rendertype $=$ abstract (accessed February 23, 2014).

20. Knudsen KA, Rosand J, Karluk D, Greenberg SM. Clinical diagnosis of cerebral amyloid angiopathy: validation of the Boston criteria. Neurology. 2001;56(4):537-539. http://www.ncbi.nlm. nih.gov/pubmed/11222803 (accessed March 17, 2014).

21. Russo MJ, Gustafson D, Vázquez S, et al. Creation of the Argentina-Alzheimer's disease neuroimaging initiative. Alzheimers Dement. 2014;10(1):S84-S87. http://linkinghub.elsevier.com/ retrieve/pii/S1552526013029166 (accessed March 14, 2014).

22. Albert MS, DeKosky ST, Dickson D, et al. The diagnosis of mild cognitive impairment due to Alzheimer's disease: recommendations from the National Institute on Aging-Alzheimer's Association workgroups on diagnostic guidelines for Alzheimer's disease. Alzheimers Dement. 2011;7(3):270-279. http://www. pubmedcentral.nih.gov/articlerender.fcgi?artid=3312027\&tool= pmcentrez\&rendertype $=$ abstract (accessed November 8, 2013).

23. Koivunen J, Scheinin N, Virta JR, et al. Amyloid PET imaging in patients with mild cognitive impairment: a 2-year follow-up study. Neurology. 2011;76(12):1085-1090. http://www.ncbi.nlm. nih.gov/pubmed/21325653 (accessed June 8, 2014). 
24. Dubois B, Feldman HH, Jacova C, et al. Revising the definition ofAlzheimer's disease: a new lexicon. Lancet Neurol. 2010; 9(11):1118-1127. http://www.ncbi.nlm.nih.gov/pubmed/20934914 (accessed February 19, 2014).

25. Summary E. The benefits of early diagnosis and intervention World Alzheimer Report 2011; 2011.

26. Yang L, Rieves D, Ganley C. Brain amyloid imaging-FDA approval of florbetapir F18 injection. $N$ Engl J Med. 2012; 367(10):885-887. http://www.ncbi.nlm.nih.gov/pubmed/22931256 (accessed March 15, 2014).

27. Clark CM, Schneider JA, Bedell BJ, et al. Use of florbetapir-PET for imaging beta-amyloid pathology. JAMA. 2011;305(3):275-283. http://www.ncbi.nlm.nih.gov/pubmed/21245183 (accessed February 19, 2014).

28. Klunk WE. Amyloid imaging as a biomarker for cerebral $\beta$-amyloidosis and risk prediction for Alzheimer dementia. Neurobiol Aging. 2011;32(suppl 1):S20-S36. http://www.pubmedcentral.nih. gov/articlerender.fcgi? artid $=3233688 \&$ tool $=$ pmcentrez\&render type $=$ abstract $($ accessed February 28, 2014).

29. Cairns NJ, Ikonomovic MD, Benzinger T, et al. Absence of Pittsburgh compound $\mathrm{B}$ detection of cerebral amyloid beta in a patient with clinical, cognitive, and cerebrospinal fluid markers of Alzheimer disease: a case report. Arch Neurol. 2009;66(12): 1557-1562. http://www.pubmedcentral.nih.gov/articlerender.fcgi? artid $=2796200 \&$ tool $=$ pmcentrez $\&$ rendertype $=$ abstract $\quad$ (accessed March 9, 2014).

30. Piguet O, Halliday GM, Creasey H, Broe GA, Kril JJ. Frontotemporal dementia and dementia with Lewy bodies in a casecontrol study of Alzheimer's disease. Int Psychogeriatr. 2009; 21(4):688-695. http://www.ncbi.nlm.nih.gov/pubmed/19493380 (accessed March 9, 2014).

31. Harms M, Benitez BA, Cairns N, et al. C9orf72 hexanucleotide repeat expansions in clinical Alzheimer disease. JAMA Neurol. 2013;70(6):736-741. http://www.pubmedcentral.nih.gov/article render.fcgi? artid $=3681841 \&$ tool $=$ pmcentre $\& \&$ rendertype $=$ abstract (accessed December 3, 2014).

32. Warren JD, Fletcher PD, Golden HL. The paradox of syndromic diversity in Alzheimer disease. Nat Rev Neurol. 2012;8(8):451-464. http://www.ncbi.nlm.nih.gov/pubmed/22801974 (accessed November 7, 2013).

33. Rascovsky K, Hodges JR, Kipps CM, et al. Diagnostic criteria for the behavioral variant of frontotemporal dementia (bvFTD): current limitations and future directions. Alzheimer Dis Assoc Disord. 2007;21(4):S14-S18. http://www.ncbi.nlm.nih.gov/ pubmed/18090417.

34. Johnson JK, Head E, Kim R, Starr A. Clinical and pathological evidence for a frontal variant of Alzheimer disease. Arch Neurol. 1999;56(10):1233-1239.

35. Grossman M. Primary progressive aphasia: clinicopathological correlations. Nat Rev Neurol. 2010;6(2):88-97. http://www. pubmedcentral.nih.gov/articlerender.fcgi?artid=3637977\&tool= pmcentrez\&rendertype $=$ abstract $($ accessed November 7, 2013).

36. Gorno-Tempini ML, Hillis AE, Weintraub S, et al. Classification of primary progressive aphasia and its variants. Neurology.
2011;76(11):1006-1014. http://www.pubmedcentral.nih.gov/ articlerender.fcgi? artid $=3059138 \&$ tool $=$ pmcentrez $\&$ rendertype $=$ abstract (accessed February 26, 2014).

37. Gorno-Tempini ML, Dronkers NF, Rankin KP, et al. Cognition and anatomy in three variants of primary progressive aphasia. Ann Neurol. 2004;55(3):335-346. http://www.pubmedcentral.nih.gov/ articlerender.fcgi?artid=2362399\&tool=pmcentrez\&rendertype $=$ abstract (accessed March 9, 2014).

38. Pike KE, Savage G, Villemagne VL, et al. Beta-amyloid imaging and memory in non-demented individuals: evidence for preclinical Alzheimer's disease. Brain. 2007;130(pt 11): 2837-2844. http://www.ncbi.nlm.nih.gov/pubmed/17928318 (accessed February 22, 2014).

39. Morris JC, Roe CM, Xiong C, et al. APOE predicts A $\beta$ but not tau Alzheimer's pathology in cognitively normal aging ccess. Ann Neurol. 2011;67(1):122-131.

40. Storandt M, Mintun MA, Head D, Morris JC. Cognitive decline and brain volume loss as signatures of cerebral amyloid-beta peptide deposition identified with Pittsburgh compound B: cognitive decline associated with Abeta deposition. Arch Neurol. 2009; 66(12):1476-1481.

41. Sperling RA, Aisen PS, Beckett LA, et al. Toward defining the preclinical stages of Alzheimer's disease: recommendations from the National Institute on Aging-Alzheimer's Association workgroups on diagnostic guidelines for Alzheimer's disease. Alzheimers Dement. 2011;7(3):280-292. http://www.pubmedcentral.nih.gov/ articlerender.fcgi? artid $=3220946 \&$ tool $=$ pmcentrez\&rendertype $=$ abstract (accessed November 8, 2013).

42. Desikan RS, McEvoy LK, Thompson WK, et al. Amyloid- $\beta$ associated clinical decline occurs only in the presence of elevated P-tau. Arch Neurol. 2012;69(6):709-713. http://www. pubmedcentral.nih.gov/articlerender.fcgi?artid=3423526\&tool= pmcentrez\&rendertype $=$ abstract (accessed June 8, 2014).

43. Gomperts SN, Rentz DM, Moran E, et al. Imaging amyloid deposition in Lewy body diseases. Neurology. 2008;71(12): 903-910. http://www.pubmedcentral.nih.gov/articlerender.fcgi? artid $=2637553 \&$ tool $=$ pmcentrez\&rendertype $=$ abstract (accessed January 21, 2014).

44. Burrell JR, Hornberger M, Villemagne VL, Rowe CC, Hodges JR. Clinical profile of PiB-positive corticobasal syndrome. PLoS One. 2013;8(4):e61025. http://www.pubmedcentral.nih.gov/article render.fcgi? artid $=3618463 \&$ tool $=$ pmcentrez\&rendertype $=$ abstract (accessed February 24, 2014).

45. Hassan A, Whitwell JL, Josephs KA. The corticobasal syndrome-Alzheimer's disease conundrum. Expert Rev Neurother. 2011;11(11):1569-1578. http://www.pubmedcentral.nih.gov/ articlerender.fcgi? artid $=3232678 \&$ tool $=$ pmcentrez\&rendertype $=$ abstract (accessed March 15, 2014).

46. Fleisher AS, Chen K, Liu X, et al. Using positron emission tomography and florbetapir F18 to image cortical amyloid in patients with mild cognitive impairment or dementia due to Alzheimer disease. Arch Neurol. 2011;68(11):1404-1411. http:// www.ncbi.nlm.nih.gov/pubmed/21747008 (accessed March 9, 2014). 